\title{
Spatial and temporal genetic structure in chloroplast and allozyme markers in Phacelia dubia implicate genetic drift
}

\author{
FOSTER LEVY* \& CHRISTOPHER L. NEAL \\ Department of Biological Sciences, East Tennessee State University, Johnson City, TN 37614, U.S.A.
}

\begin{abstract}
For neutral genes, uniparental inheritance is expected to reduce effective population size relative to biparentally inherited genes. In finite populations, the ensuing genetic drift can cause stronger spatial and temporal differentiation. An intrapopulation polymorphism in chloroplast DNA was used to examine relative spatial and temporal population structure of chloroplast and allozyme markers in the annual plant Phacelia dubia. There was significant differentiation among populations at chloroplast markers but not for allozyme loci. A fine-scale analysis showed significant structure among sites within populations for chloroplast markers and local heterozygote deficiencies at allozyme loci. These spatial analyses suggest that gene flow via pollen exceeds that via seed. Temporal variation in chloroplast markers, assessed over a 10-year period, was evident in two of four populations, and allozyme loci were characterized by temporal variation in rare-allele frequencies. Population structure appeared to be related to the intensity and type of human disturbance influencing each population. Habitat destruction promoted isolation and enhanced differentiation, whereas mowing increased seed dispersal and reduced differentiation for chloroplast markers. At this time, genetic drift appears to be the primary force shaping chloroplast gene frequencies.
\end{abstract}

Keywords: chloroplast DNA, gene flow, genetic drift, Phacelia, spatial structure, temporal structure.

\section{Introduction}

In finite populations, genetic drift may be the predominant force governing allele frequencies of neutral loci. Drift effects include an intrapopulation temporal component, that is populations diverge from an initial state, and a spatial dimension in which a series of replicate populations diverge from each other. For neutral genetic markers, spatial differentiation among populations, estimated by $F_{\mathrm{ST}}$, provides an indicator of the cumulative effects of drift and migration. Because effective population size for maternally inherited organelles $\left(N_{\mathrm{e} \text {,mat }}\right)$ is expected to be one-half or less than that for nuclear genes $\left(N_{\mathrm{e}, \mathrm{nuc}}\right)$, and random packaging of genomes into gametes rather than formal apportionment appears to govern chloroplast transmission patterns, genetic drift is expected to play a stronger role in governing frequencies of organelle genes compared to nuclear genes (Birky, 1983). The resultant prediction of stronger population differentiation for maternally inherited genes $\left(F_{\text {STmat }}\right)$ relative to nuclear genes $\left(F_{\text {STnuc }}\right)$

*Correspondence. E-mail: levyf@etsu.edu has been borne out for mitochondrial DNA markers compared to nuclear-encoded markers in animals such as Hawaiian Drosophila (DeSalle et al., 1987) and brown trout (Hansen \& Loeschcke, 1996). In both species, population bottlenecks were believed to have exacerbated drift effects. Chloroplast markers in several flowering plants also show substantially greater differentiation than nuclear markers (Ennos, 1994; McCauley et al., 1996).

More pronounced temporal variation in animal mitochondrial genes compared to nuclear genes is also consistent with differences in effective population sizes for the two genomes (DeSalle et al., 1987; Hansen \& Loeschcke, 1996). Animal mitochondrial and flowering plant chloroplast transmission share uniparental inheritance, high genome copy number per organelle and apparent random sorting of genomes and organelles during gamete formation, but limited intraspecific polymorphisms in chloroplast DNA have hindered both temporal and fine-scale spatial analyses in plants. Recent studies have uncovered species that harbour substantial amounts of chloroplast DNA variation, the majority of which resides between rather than within populations (Milligan, 1991; Hong et al., 1993; Levy 
et al., 1996). Combined analyses of spatial structuring at nuclear- and chloroplast-encoded loci in flowering plants show greater structure in the latter, which suggests gene flow via pollen exceeds that from seeds (reviewed in Ennos, 1994; Levy et al., 1996; McCauley, 1997). In conifers, where chloroplast DNA is paternally inherited and mitochondrial DNA is maternally inherited, it is the latter which shows greater population differentiation, again suggesting restricted seed movement compared to pollen dispersal (Latta \& Mitton, 1997).

Mating systems can influence drift and migration through their effects on effective population size and gamete dispersal. Because plant mating systems tend to be more diverse, within and between taxa, than those of complex multicellular animals, generalizations concerning the scale of spatial structuring and the extent of temporal variation of animal mitochondrial genes are not readily applicable to chloroplast genes. In this study, we assessed temporal and fine-scale spatial structure in the annual plant Phacelia dubia (L.) Fern. Phacelia dubia has a mixed mating system of selfing and outcrossing with populations consisting of temporally stable patches (del Castillo, 1994). Halictid and andrenid bees are the primary pollinators. Most seeds germinate in the first year but some may persist in the soil for up to seven years (Baskin \& Baskin, 1973). Both population bottlenecks and asymmetrical seed and pollen dispersal have been documented in the species (del Castillo, 1994). Moreover, both forms of a chloroplast DNA polymorphism co-occurred in several populations surveyed by Levy et al. (1996). Because some chloroplast haplotypes were both restricted to particular populations and common or fixed within those populations, Levy et al. (1996) suggested that interpopulation, long-distance seed dispersal was rare. In species where gene flow via pollen exceeds gene flow via seeds, fine-scale spatial differentiation for maternally inherited genes may also develop within populations and exceed that for biparentally inherited markers (McCauley et al., 1996). To test for fine-scale differentiation, we used an analysis of chloroplast DNA and allozyme gene frequencies to compare spatial differentiation among local sites within populations.

Although genetic drift is a time-dependent process, there has been virtually no attempt to measure variation in chloroplast gene frequencies over time. Intrapopulation plastome and allozyme polymorphisms in P. dubia provide an opportunity to assess temporal variation directly, with the expectation that $F_{\text {STmat }}>F_{\text {STnuc }}$ within populations. This study represents the first analysis of long-term population dynamics for chloroplast markers in populations composed of completely interfertile individuals.

\section{Methods}

\section{Plastome polymorphism}

Two major plastome lineages within the annual plant Phacelia dubia were uncovered in an intraspecific survey of chloroplast DNA variation (Levy et al., 1996). These lineages (designated plastome types D and G) differed by a suite of six restriction fragments, five of which represent base-pair substitutions and one probable length variant. Variation among plastomes D and $G$ was detected with each of six restriction enzymes and variable fragments were scattered throughout the genome. The physically dispersed nature of the variation within genomes suggests that the split in the major plastome lineages is not the result of a single genome rearrangement but is more likely characterized by a series of independent mutation events. In light of the relatively conserved nature of the chloroplast genome, the $\mathrm{D}$ and $\mathrm{G}$ lineages probably represent an ancient split in the plastid phylogeny. An initial survey conducted on samples collected in 1987 showed that plastomes D and $\mathrm{G}$ coexisted in each of four populations of the narrow endemic $P$. dubia var. georgiana, and in one of three populations of the more widespread, but allopatric, P. dubia var. dubia.

\section{Population descriptions}

Phacelia dubia var. georgiana is endemic to granite outcrops in the piedmont region of Georgia and adjacent Alabama. Plastome type was determined for samples of plants from each of three populations of $P$. dubia var. georgiana: Echol's Mill (EM) (Oglethorpe Co., GA), the type locality of P. dubia var. georgiana, consists of a mix of outcrop and forest patches that extends over $3 \mathrm{~km}$. The EM location encompasses active granite quarries with extensive local disturbance that results in complete destruction of some outcrop habitat. At Echol's Mill, the 'road' subpopulation (EM-Rd) is bordered by an unpaved road and forest patches. It is a small area of $\approx 30 \mathrm{~m}$ in diameter that is undergoing succession from a plant cover dominated by lowgrowing herbs to one dominated by perennial grasses. The 'river' subpopulation at Echol's Mill (EM-Rv), also $\approx 30 \mathrm{~m}$ in diameter, is located $2 \mathrm{~km}$ from the road subpopulation and is bordered by a small stream, an unpaved road and active quarry operations. The Appling population (AP) (Columbia Co., GA.) occupies a relatively small (diameter of less than $100 \mathrm{~m}$ ) granite outcrop that is bordered by a paved road, a lawn and forest. A power line crosses over the portion of the outcrop that adjoins the road. The power line right-ofway is regularly mowed. Kiokee Creek outcrop (KIO) is 
the most pristine - it is located only $3 \mathrm{~km}$ from the Appling outcrop but it is completely surrounded by relatively undisturbed forest. The outcrop is $\approx 250 \mathrm{~m}$ in diameter with islands of shrub thickets on the outcrop. Thus, two pairs of samples (EM-Rd/EM-Rv; AP/KIO) were from areas in relatively close geographical proximity, but the EM and $\mathrm{AP} / \mathrm{KIO}$ regions are separated by $\approx 75 \mathrm{~km}$. Population sizes vary from fewer than one hundred plants maturing seeds in poor years to more than a thousand plants in favourable years.

\section{Population samples}

Plastome frequencies were assayed over an eight-year period in each population. Spatial structure was assessed at the local (within population) and geographical (between populations) scales, but the detailed sampling necessary for local analyses was carried out only during 1992 and 1994. Seed samples from 1987 and 1990 were collected from random locations throughout each population, but during these years no attempt was made to identify the collection location of plants from which seeds were collected. Seeds that were grown and the resultant plants that were assayed were chosen randomly but each was derived from a different maternal parent. On granite outcrops, individuals of $P$. dubia var. georgiana are not scattered throughout the area, but are restricted to patches or areas we term local sites. To assess the degree of local differentiation within populations, in 1992 and 1994, four to seven sites within each population were chosen to encompass the spectrum of spatial and biological diversity. At each local site, seeds were collected from plants within a $1 \mathrm{~m}^{2}$ area. There was no attempt to collect from nearest neighbours or to quantify distances between individual plants, because the weak stems of many plants often lead to intertwining of individuals, and the annual habit means that spatial relationships among individuals will not persist beyond one year.

\section{DNA isolation and detection}

All DNA samples were extracted from plants grown from field-collected seeds. For 1987 samples, cpDNA was isolated using procedures outlined in Levy et al. (1996). Plastome type was assayed visually by ethidium bromide staining of purified cpDNA, and for some samples plastome types were confirmed by Southern hybridizations of diagnostic restriction fragments (Levy et al., 1996). Genomic DNA was isolated from all other plants by incubating leaf extracts for $30 \mathrm{~min}$ in hot $\left(65^{\circ} \mathrm{C}\right)$ CTAB buffer followed by two chloroformisoamyl alcohol extractions. DNA was precipitated in isopropanol, and the resultant pellet was washed in $70 \%$ ethanol and resuspended in TE. Genomic DNAs were treated with RNase and digested with either AvaII or its isoschizomer, $\operatorname{Sin} I$. These restriction enzymes reveal two plastome-diagnostic RFLPs; a D plastome-specific $9.0 \mathrm{~kb}$ fragment is digested to $5.3 \mathrm{~kb}$ and $4.7 \mathrm{~kb}$ fragments in plastome $\mathrm{G}$ and another $\mathrm{D}$ plastome-specific $2.4 \mathrm{~kb}$ fragment is cut into $2.1 \mathrm{~kb}$ and $0.3 \mathrm{~kb}$ fragments in plastome G (Levy et al., 1996). Resultant genomic DNA digests were separated on $0.8 \%$ agarose gels, Southern blotted and hybridized to a homologous digoxigenin-labelled probe consisting of restrictiondigested total chloroplast DNA. DNA samples were scored as either the D or G plastome from autoradiographs.

\section{Statistical analyses}

The subdivision parameter for chloroplast markers, $F_{\text {STmat }}$, was estimated by the AMOVA procedure using ARLEQUIN software (Schneider et al., 1997). Differentiation was considered significant when: (i) the $P$-value of a permutation test $(10000$ permutations using ARLEQUIN) was less than 0.05; and (ii) two times the standard error derived from a jackknife estimate of the standard deviation of $F_{\mathrm{ST}}$ (software provided by B. Weir) did not overlap with zero.

\section{Allozyme analyses}

To estimate the level of geographical differentiation among populations at nuclear-encoded loci $\left(F_{\text {STnuc }}\right)$, population samples from seeds collected in 1986 were assayed for allozyme variation at 11 loci $(A L D, G O T 1$, GOT2, MDH, ME, PGI1, PGI2, PGM, PRO1, SDH, $S O D$ ) using eight different enzyme systems. Sample sizes per locus within the three populations ranged from 5376 for AP, 34-43 for KIO and 66-72 for EM. Detailed tissue extraction and electrophoresis methods are presented in Levy (1989). Gene diversity summary statistics including heterozygosity were estimated from data on all 11 loci using GENESTRUT software (Constantine et al., 1994). Single-locus estimates of $F_{\text {STnuc }}$ were used to test for local differentiation using GENEPOP (Raymond \& Rousset, 1995a).

To test for temporal variation in allele frequencies, two allozyme loci $(G O T 1$, GOT2) that showed intrapopulation polymorphism were analysed using data from 1986 and 1992 at KIO; the temporal analyses for EM also included data from 1994. For single-locus tests of temporal differentiation, GENEPOP (Raymond \& Rousset, 1995a) was used to estimate $F_{\text {STnuc }}$ among years within populations. Markov chain methods were applied to contingency tables consisting of years in columns and alleles (or genotypes) in rows, with allele 
(or genotype) frequencies as entries to test the significance of potential allelic and genotypic differentiation, respectively (Raymond \& Rousset, 1995b).

To summarize the sampling scheme, prior to 1992, samples for both the allozyme and chloroplast analyses were chosen randomly. Samples from 1992 and 1994 were delineated by local sites within each population, and at EM the river and road areas were treated as separate subpopulations.

\section{Results}

\section{Plastome variation}

Local spatial patterning within populations Most sites within AP supported a mix of plants with plastomes D and G during both 1992 and 1994 (Table 1). In 1992, the frequency of plastome D varied among sites from zero to 0.86 (Table 1) which led to significant spatial substructuring $\left(F_{\mathrm{STmat}}=0.24\right.$; Table $\left.2 \mathrm{a}\right)$. Although the range in plastome frequencies was smaller in 1994 (Table 1), the heterogeneity in plastome frequencies among sites was again significant (Table 2a) but the degree of subdivision was lower $\left(F_{\mathrm{STmat}}=0.16\right)$. At KIO, in both the 1992 and 1994 samples, $F_{\text {STmat }}$ was relatively high and these differences in plastome frequencies among sites were significant for both sample years (Table 2a). Unlike AP, sites within KIO tended to be dominated by one plastome type (Table 1). Extensive substructuring at KIO was exemplified by one site that was fixed for plastome $G$, the minority type in that population (Table 1), with a neighbouring site fixed for plastome D.

The most extreme population subdivision was noted at EM, where the river site was fixed for plastome D in both 1992 and 1994, but where only $2 \mathrm{~km}$ distant, at the road site, plastome $\mathrm{D}$ was found in only one of 39 plants (Table 1). With plastome frequencies near fixation at each subpopulation in 1992, there was obviously no significant heterogeneity among sites within either subpopulation. The rarity within and among sites suggests plastome D probably existed as a rare variant in the road subpopulation rather than as a locally common minority in 1992, but by 1994, plastome D had spread so that the population frequency of plastome $\mathrm{D}$ increased and its presence was detected at three of four sample sites.

Geographical differentiation among populations Differences in plastome frequencies among populations provided evidence of significant large-scale geographical heterogeneity during two of the four years $(1992,1994)$ sampled (Table 3). Differentiation in 1987 must be considered marginal because of a lower estimate of $F_{\text {STmat }}$ compared to later years and because two standard errors of the jackknife estimate of $F_{\mathrm{STmat}}$ overlapped zero (Table 3). Pairwise estimates of spatial differentiation among population pairs in close proximity showed that the large-scale effect was not caused by differences arising from a distance effect (Table 2c). Rather, comparisons among population pairs in close proximity were all significant (Table 2c). Extreme plastome frequency differences among the two subpopulations at EM indicate that more proximal populations do not necessarily show more similar frequencies.

Temporal patterns within populations Evidence for temporal fluctuations was suggestive in the AP population (the permutation, but not the jackknife, test was significant) compared to relative temporal stability at KIO (Tables 4 and 2b). At AP the degree of temporal

Table 1 Spatial patterns of plastome occurrence within populations of Phacelia dubia. Frequency $(p)$ of plastome D among sites within each of three populations for 1992 and 1994. Each row represents a different site within a population. Sites within years arranged in ascending frequency of plastome D but sites on the same line do not represent the same sample locations across years. $n$, sample size

\begin{tabular}{|c|c|c|c|c|c|c|c|c|c|c|c|c|c|c|c|}
\hline & & & & & & & & \multicolumn{8}{|c|}{ EM } \\
\hline \multicolumn{4}{|c|}{ AP } & \multicolumn{4}{|c|}{ KIO } & \multicolumn{4}{|c|}{$\mathrm{Rv}$} & \multicolumn{4}{|c|}{$\mathrm{Rd}$} \\
\hline \multicolumn{2}{|c|}{1992} & \multicolumn{2}{|c|}{1994} & \multicolumn{2}{|c|}{1992} & \multicolumn{2}{|c|}{1994} & \multicolumn{2}{|c|}{1992} & \multicolumn{2}{|c|}{1994} & \multicolumn{2}{|c|}{1992} & \multicolumn{2}{|c|}{1994} \\
\hline$p$ & $n$ & $p$ & $n$ & $p$ & $n$ & $p$ & $n$ & $p$ & $n$ & $p$ & $n$ & $p$ & $n$ & $p$ & $n$ \\
\hline 0.00 & 9 & 0.00 & 10 & 0.00 & 7 & 0.27 & 11 & 1.00 & 6 & 1.00 & 9 & 0.00 & 2 & 0.00 & 5 \\
\hline 0.25 & 8 & 0.17 & 12 & 0.33 & 3 & 0.92 & 13 & 1.00 & 7 & 1.00 & 15 & 0.00 & 6 & 0.25 & 4 \\
\hline 0.40 & 10 & 0.27 & 11 & 1.00 & 1 & 1.00 & 8 & 1.00 & 7 & 1.00 & 20 & 0.00 & 7 & 0.67 & 3 \\
\hline 0.50 & 4 & 0.45 & 11 & 1.00 & 3 & 1.00 & 10 & 1.00 & 9 & & & 0.00 & 8 & 1.00 & 1 \\
\hline 0.50 & 6 & 0.58 & 12 & 1.00 & 3 & & & 1.00 & 10 & & & 0.00 & 9 & & \\
\hline \multirow{2}{*}{0.86} & 7 & & & 1.00 & 4 & & & & & & & 0.14 & 7 & & \\
\hline & & & & 1.00 & 14 & & & & & & & & & & \\
\hline
\end{tabular}


Table 2 Estimates of Phacelia dubia population subdivision at chloroplast loci $\left(F_{\mathrm{STmat}}\right)$ with two indicators of significance: two times the jackknife estimate of standard errors, and probabilities associated with permutation tests for heterogeneity: (a) among sites within populations; (b) among years within populations; (c) among pairs of populations in close proximity. $N$, number of populations or sites within populations; NA, not applicable because jackknife estimates are not possible when comparing two populations

\begin{tabular}{|c|c|c|c|c|c|}
\hline Population & Year(s) & $N$ & $F_{\text {STmat }}$ & $2 \times \mathrm{SE}$ & Permutation \\
\hline \multicolumn{6}{|c|}{ (a) Among sites within populations } \\
\hline AP & 1992 & 6 & 0.24 & 0.20 & 0.01 \\
\hline AP & 1994 & 5 & 0.16 & 0.12 & 0.02 \\
\hline KIO & 1992 & 7 & 0.90 & 0.19 & $<0.001$ \\
\hline $\mathrm{KIO}$ & 1994 & 4 & 0.59 & 0.49 & $<0.001$ \\
\hline EM-Rd & 1992 & 6 & -0.02 & 0.00 & 0.20 \\
\hline EM-Rd & 1994 & 4 & 0.38 & 0.52 & 0.08 \\
\hline \multicolumn{6}{|c|}{ (b) Among years within populations } \\
\hline AP & 1987-1994 & 4 & 0.16 & 0.24 & 0.002 \\
\hline $\mathrm{KIO}$ & 1987-1994 & 4 & -0.03 & 0.01 & 0.91 \\
\hline EM-Rv & 1992-1994 & 2 & 0.00 & NA & NA \\
\hline EM-Rd & 1992-1994 & 2 & 0.32 & NA & 0.001 \\
\hline \multicolumn{6}{|c|}{ (c) Among population pairs in close proximity } \\
\hline $\mathrm{AP} / \mathrm{KIO}$ & 1992 & 2 & 0.23 & NA & 0.001 \\
\hline $\mathrm{AP} / \mathrm{KIO}$ & 1994 & 2 & 0.36 & NA & $<0.001$ \\
\hline $\mathrm{EM}: \mathrm{Rv} / \mathrm{Rd}$ & 1992 & 2 & 0.97 & NA & $<0.001$ \\
\hline EM: $\mathrm{Rv} / \mathrm{Rd}$ & 1994 & 2 & 0.83 & NA & $<0.001$ \\
\hline
\end{tabular}

Table 3 Yearly estimates of chloroplast differentiation among populations of Phacelia dubia ( $F_{\text {STmat }}$, with jackknife estimates of standard errors and probabilities associated with permutation tests. $N$, number of populations

\begin{tabular}{llrcc}
\hline Year & $N$ & $F_{\text {STmat }}$ & $2 \times \mathrm{SE}$ & Permutation \\
\hline 1987 & 3 & 0.23 & 0.32 & 0.05 \\
1990 & 3 & -0.09 & 0.02 & 0.78 \\
1992 & 4 & 0.60 & 0.29 & $<0.001$ \\
1994 & 4 & 0.47 & 0.18 & $<0.001$ \\
\hline
\end{tabular}

subdivision was not high $\left(F_{\mathrm{STmat}}=0.16\right)$ but the permutation test suggests significant changes in plastome frequencies over time. Because of the extreme plastome frequency differentiation between the river and road subpopulations at EM, temporal variation was only considered for the two years $(1992,1994)$ for which subpopulation data were available. Plastome frequencies were stable at EM-Rv where plastome D remained near fixation (Table 4). In contrast, EM-Rd experienced a significant increase in plastome D over the two-year time period (Table $2 b$ ). The widely divergent plastome

Table 4 Temporal patterns of plastome occurrence. Frequency $(p)$ of plastome D in Phacelia dubia populations in each of four years. River $(\mathrm{Rv})$ and road $(\mathrm{Rd})$ subpopulations within EM were not distinguished prior to 1992. $n$, sample size

\begin{tabular}{|c|c|c|c|c|c|c|c|c|c|c|}
\hline \multirow{2}{*}{ Year } & \multicolumn{10}{|c|}{ Population } \\
\hline & \multicolumn{2}{|c|}{ AP } & \multicolumn{2}{|c|}{$\mathrm{KIO}$} & \multicolumn{6}{|c|}{ EM } \\
\hline 1987 & 0.25 & 8 & 0.67 & 9 & & & 0.13 & 8 & & \\
\hline 1990 & 0.91 & 11 & 0.88 & 8 & & & 0.93 & 14 & & \\
\hline 1992 & 0.39 & 44 & 0.74 & 35 & 1.00 & 39 & & & 0.03 & 39 \\
\hline 1994 & 0.30 & 56 & 0.79 & 42 & 1.00 & 44 & & & 0.31 & 13 \\
\hline
\end{tabular}

(C) The Genetical Society of Great Britain, Heredity, 82, 422-431. 
frequencies between the 1987 and 1990 samples at EM (Table 4) must be viewed with caution. Prior to 1992, samples from the two subpopulations were not treated separately, hence the variation in plastome frequency among these two samples may simply reflect the effects of small sample sizes, or they may result from biased selections of seeds for collection, germination and growth.

\section{Allozyme variation}

Allozyme spatial patterns At the broad geographical scale, allozymes showed a virtual absence of differentiation among populations $\left(F_{\text {STnuc }}=0.02\right)$. In fact, at each of the 11 loci surveyed the most common allele was the same in each of the three populations (Table 5). Moreover, the frequency of these common alleles exceeded 0.90 at all loci in all populations, with the exception of one locus each in AP and KIO. In spite of the shared common alleles, only three loci ( $A L D, P R O 1$, $S O D)$ were monomorphic across populations. The allozyme profile in these populations can best be characterized as one in which a common allele is geographically widespread, but populations differ in the identity of rare alleles. As a consequence of these Ushaped allele-frequency spectra, average heterozygosity was relatively low $(\leq 0.06)$ within each population (Table 5), and this may have limited our ability to detect significant trends (Leonardi \& Menozzi, 1996).

After pooling genotypic classes with expectations of five or fewer individuals, none of the allozyme loci in any of the three populations showed significant deviations from Hardy-Weinberg expectations. Nevertheless, the hierarchical analysis showed evidence of nonrandom mating $\left(F_{\mathrm{IS}}=0.22\right)$ within populations, and deviations at individual loci were invariably skewed towards excesses of homozygotes and deficiencies of heterozygotes. This pattern, suggestive of inbreeding, is not unexpected for a self-compatible annual plant with no specialized seed-dispersal mechanism.

Because seed collections prior to 1992 were not delineated by sites within populations, an analysis of spatial variation at the local level was not possible from the 1986 allozyme data. Furthermore, the detection of spatial structure is difficult when heterozygosity or sample size is low (Leonardi \& Menozzi, 1996) as was the case for GOT1 and GOT2 in both the 1992 and 1994 samples from EM and for GOT1 in the 1992 sample from KIO (Table 6). However, in the 1992 sample from KIO, GOT2 alleles $a$ and $b$ were present at frequencies of 0.84 and 0.16 , respectively. The less frequent $b$ allele was absent from four of the seven sites and present at frequencies of $0.20,0.27$ and 0.33 at the remaining three sites. Subdivision was evident at this locus $\left(F_{\mathrm{IT}}=0.34\right)$,
Table 5 Allele frequencies at 11 allozyme loci in three populations of Phacelia dubia. Sample sizes per locus: AP (53-76); KIO (27-43); EM (56-71). Average expected heterozygosity $\left(\mathrm{Av} . H_{\mathrm{s}}\right.$ ) and average number of alleles per locus $(A / L)$ are shown for each population

\begin{tabular}{|c|c|c|c|c|}
\hline \multirow[b]{2}{*}{ Locus } & \multirow[b]{2}{*}{ Allele } & \multicolumn{3}{|c|}{ Population } \\
\hline & & AP & $\mathrm{KIO}$ & EM \\
\hline$A L D$ & $a$ & 1.0 & 1.0 & 1.0 \\
\hline \multirow[t]{4}{*}{ GOT1 } & $b$ & - & 0.02 & - \\
\hline & $c$ & 1.0 & 0.94 & 0.96 \\
\hline & $d$ & - & - & 0.03 \\
\hline & $e$ & - & 0.04 & - \\
\hline \multirow{3}{*}{ GOT2 } & $a$ & 0.98 & 0.79 & 1.0 \\
\hline & $b$ & 0.01 & 0.17 & - \\
\hline & $c$ & 0.01 & 0.04 & - \\
\hline \multirow[t]{4}{*}{$M D H$} & $a$ & 0.99 & 1.0 & 0.95 \\
\hline & $b$ & - & - & 0.01 \\
\hline & $e$ & 0.01 & - & - \\
\hline & $f$ & - & - & 0.04 \\
\hline \multirow[t]{2}{*}{$M E$} & $a$ & 1.0 & 1.0 & 0.99 \\
\hline & $b$ & - & - & 0.01 \\
\hline \multirow[t]{4}{*}{$P G I 1$} & $a$ & - & 0.01 & - \\
\hline & $b$ & 0.91 & 0.98 & 0.98 \\
\hline & $c$ & 0.01 & - & - \\
\hline & $d$ & 0.08 & 0.01 & 0.02 \\
\hline \multirow[t]{2}{*}{ PGI2 } & $a$ & 1.0 & 1.0 & 0.99 \\
\hline & $c$ & - & - & 0.01 \\
\hline \multirow[t]{2}{*}{$P G M$} & $a$ & 0.98 & 0.99 & 1.0 \\
\hline & $c$ & 0.02 & 0.01 & - \\
\hline PROI & $a$ & 1.0 & 1.0 & 1.0 \\
\hline \multirow[t]{3}{*}{$S D H$} & $a$ & 0.84 & 0.92 & 0.91 \\
\hline & $b$ & 0.11 & 0.07 & 0.09 \\
\hline & $c$ & 0.05 & 0.01 & - \\
\hline$S O D$ & $a$ & 1.0 & 1.0 & 1.0 \\
\hline Av. $H_{\mathrm{s}}$ & & 0.05 & 0.06 & 0.04 \\
\hline$A / L$ & & 1.73 & 1.82 & 1.64 \\
\hline
\end{tabular}

but it was caused by inbreeding within sites $\left(F_{\mathrm{IS}}=0.37\right)$ rather than nonrandom mating among sites $\left(F_{\mathrm{ST}}=-0.04\right)$. A test of a site $\times$ allele contingency table also revealed heterogeneity in allele frequencies among sites $\left(G_{6}=16.9 ; P<0.01\right)$.

Because there was evidence of spatial structuring for both nuclear and cytoplasmically transmitted genes at KIO in 1992, a test for nonrandom associations among alleles of the chloroplast and nuclear genomes was conducted. A $2 \times 3$ (two plastome types; three genotypes at GOT2) contingency test revealed no evidence of cytonuclear disequilibrium between plastome type and GOT2 genotype $\left(G_{2}=5.6 ; P=0.07\right)$, but there was evidence of a plastome-genic association; GOT2 allele $b$ was found only in association with plastome D $\left(G_{1}=6.72 ; P<0.01\right)$. 
Table 6 Allele frequencies at GOT1 and GOT2 for each sampling year in two populations of Phacelia dubia, Echol's Mill $(\mathrm{EM})$ and Kiokee Creek $(\mathrm{KIO})$. Estimates of temporal differentiation $\left(F_{\mathrm{STnuc}}\right)$ are shown with $P$ values associated with genotypic and allelic differentiation. $n$, sample size; NA, not sampled. Dashes indicate the absence of an allele from a sample

\begin{tabular}{|c|c|c|c|c|c|c|c|c|}
\hline \multirow[b]{3}{*}{ Population } & & & \multicolumn{3}{|c|}{ Allele frequencies } & \multicolumn{3}{|c|}{ Temporal effects } \\
\hline & & & & Year & & & $P$ val & \\
\hline & \multicolumn{2}{|c|}{ Locus/Allele } & $1986(n)$ & $1992(n)$ & $1994(n)$ & $F_{\text {STnuc }}$ & Genotypic & Allelic \\
\hline \multirow[t]{3}{*}{$\mathrm{EM}$} & GOT1 & $b$ & $-(72)$ & $-(25)$ & $0.04(14)$ & 0.01 & 0.42 & 0.24 \\
\hline & & $c$ & 0.03 & 0.04 & 0.11 & & & \\
\hline & & $d$ & 0.96 & 0.96 & 0.86 & & & \\
\hline \multirow[t]{2}{*}{ EM } & GOT2 & $a$ & $1.00(71)$ & $0.96(25)$ & 0.93 (14) & 0.05 & 0.48 & 0.05 \\
\hline & & $b$ & - & 0.04 & 0.07 & & & \\
\hline \multirow{3}{*}{ KIO } & GOT1 & $b$ & $0.02(43)$ & $-(43)$ & NA & 0.03 & 0.11 & 0.06 \\
\hline & & $c$ & 0.94 & 1.00 & NA & & & \\
\hline & & $e$ & 0.04 & - & NA & & & \\
\hline \multirow[t]{3}{*}{$\mathrm{KIO}$} & GOT2 & $a$ & $0.79(43)$ & $0.84(42)$ & NA & -0.01 & 0.27 & 0.29 \\
\hline & & $b$ & 0.17 & 0.16 & NA & & & \\
\hline & & $c$ & 0.04 & - & NA & & & \\
\hline
\end{tabular}

Allozyme temporal patterns of the four tests for temporal fluctuations, there was significant allelic variation among years at GOT2 in EM and marginally significant allelic variation at GOT1 in KIO (Table 6). The former effect was caused by a progressive increase in the rare GOT2 $b$ allele over the 8-year sample period (but note decreasing samples sizes over this period), whereas the temporal effect at KIO was caused by the loss of two rare alleles from the 1992 sample. At all four allozyme loci for which temporal effects were examined, the common allele at each locus remained common throughout the sample period, and all estimates of $F_{\mathrm{ST}}$ were low (Table 6).

\section{Discussion}

\section{Limited seed dispersal contributes to genetic drift}

Estimates of population differentiation based upon combined analyses of either animal mitochondrial (DeSalle et al., 1987; Hansen \& Loeschcke, 1996) or plant chloroplast (Ennos, 1994; McCauley, 1994; Levy et al., 1996; McCauley et al., 1996) genomes are generally lower than those of nuclear loci in corresponding species. Genetic drift is the most likely factor underlying this emerging consensus that frequencies of cytoplasmically transmitted genes are more labile in space and time than biparentally transmitted nuclear genes. In Phacelia, spatial differentiation of a chloroplast marker was high, both among sites within populations as well as between populations. But there was no evidence for a relationship between geographical distance and plastome frequencies; population pairs in close proximity (AP/KIO; EM-Rd/EM-Rv) showed very different plastome frequencies among members of each pair. These data suggest that isolation by distance is sufficient to maintain maternally inherited population differences over relatively short geographical distances and that local seed dispersal with ensuing genetic drift may be the cause of population differentiation.

For neutral loci in a polymorphic population, simulations show that 10-30 generations of restricted dispersal are sufficient to generate differentiation (Epperson, 1990), with the scale of differentiation dependent upon the shape of the dispersal profile (Ibrahim et al., 1996). When limited seed dispersal is the cause of cytoplasmic differentiation, species-specific dispersal profiles should shed light on the expected scale of differentiation. Because there are no special dispersal mechanisms in $P$. dubia, most seeds disperse less than $0.5 \mathrm{~m}$ from the mother plant (del Castillo, 1994). With such limited seed dispersal, fine-scale cytoplasmic differentiation can be expected, and was observed within populations in the current study. For example, at KIO, neighbouring sites separated by only tens of metres supported dramatically different plastome assemblages. Moreover, the extreme differentiation of the two subpopulations at EM, and the relative temporal stability of the differences between these subpopulations, indicate that a distance of only a few kilometres is sufficient to maintain long-term population differentiation for chloroplast genes. Our inability to detect a biosynthetic difference among plastome types (unpubl. data) and an absence of evident environmental heterogeneity suggest that a modest seed-dispersal profile, rather than differential selection on plastomes, may be the logical cause of such small-scale differentiation. 
Unlike the plastome analysis, the allozyme analysis revealed a virtual absence of spatial differentiation among populations $\left(F_{\text {STnuc }}=0.02\right)$, but the relatively low heterozygosity that characterized each of the study populations may have limited our ability to detect differentiation (Leonardi \& Menozzi, 1996). At allozyme loci, the Phacelia populations differed primarily in the identity of their respective rare alleles, a pattern that suggests that the rare alleles represent recently generated, population-specific variation. It should also be noted that an allele-frequency spectrum characterized by low heterozygosity and an apparent excess of rare alleles is typical of the pattern expected in populations recovering from monomorphism following population bottlenecks (Maruyama \& Fuerst, 1984).

The difference in $F_{\text {STnuc }}$ and $F_{\text {STmat }}$ can be used to estimate the relative magnitude of gene flow via pollen and seeds by the expression: pollen flow/seed flow $=$ $\left[A\left(1+F_{\mathrm{IS}}\right)-2 C\right] / C$ (eqn 5a, Ennos, 1994), where $A=$ $\left(1 / F_{\text {STnuc }}-1\right)$, and $C=\left(1 / F_{\text {STmat }}-1\right)$.

For $P$. dubia, this relationship results in an estimate of gene flow via pollen that is 47.6 times greater than that for gene flow via seed dispersal. For $P$. dubia, the approximation for the relative contribution of seed dispersal to gene flow is in agreement with direct observations of more limited dispersal of seeds than pollen (del Castillo, 1994) and supports the suggestion that differences in chloroplast and nuclear population structure are biologically meaningful.

Patterns of differentiation within populations parallel those at the global level in showing relatively high $F_{\text {STmat }}$ and low $F_{\text {STnuc. }}$. For nuclear-encoded loci, local genetic structure occurs most commonly in vegetatively spreading species of fungi (Milgroom \& Lipari, 1995), plants (Hossaert-McKey et al., 1996; Caujape-Castells \& Pedrola-Monfort, 1997) and animals (Hebert \& Ward, 1976). In sexually reproducing species, local structure is common in both plants (Bonnin et al., 1996) and animals (Viard et al., 1997) that self, but structure is low in obligate outcrossers (reviewed in Heywood, 1991). Inbreeding resulting from limited pollen dispersal reduces $N_{\mathrm{e}, \text { nuc }}$ and promotes structure, but it has no effect on $N_{\mathrm{e}, \mathrm{mat}}$. Phacelia dubia has a mixed mating system that combines outcrossing with autogamy (del Castillo, 1994). In addition to del Castillo's (1994) direct demonstration of limited pollen dispersal, a nuclear allele/plastome association provided indirect evidence of local pollen dispersal within populations. In KIO, the less common GOT2 $b$ allele showed an exclusive association with plastome $\mathrm{D}$, which suggests that the nuclear-encoded allele has not spread to sites harbouring the rarer plastome G. Populations in both del Castillo's (1994) and our study were characterized by a patchy structure. Although all of these populations showed evidence of restricted pollen-mediated gene flow and inbreeding, spatial structure was quite low within populations. The relatively high $F_{\text {STmat }}$ suggests that a pollen-specific characteristic, such as occasional longdistance pollen dispersal, may be sufficient to minimize $F_{\text {STnuc }}$. The local pattern of high $F_{\text {STmat }}$ and low $F_{\text {STnuc }}$ in Phacelia was similar to observations in Silene alba, where the estimated pollen:seed dispersal differential increased with decreasing scales of analysis (McCauley, 1997). In contrast to the mixed mating system of P. dubia, S. alba is dioecious and hence an obligate outcrosser.

\section{Human factors and population structure}

The populations under study have been subject to different levels and manifestations of human-induced disturbance. For example, AP is bordered by a residence, a power line and blacktop road, all of which are maintained by mowing. In contrast, the nearby KIO population is relatively undisturbed and shows much greater within-population $F_{\text {STmat }}$ compared to AP. Mowing activity may be actively degrading $F_{\text {STmat }}$ at AP by extending seed-dispersal profiles. The lesser disturbance at KIO may account for the greater stability of plastome frequencies over time and the presence of local spatial structure in nuclear genes. Both the road and river subpopulations at EM adjoin low-usage, minimally maintained, unpaved roads, but granite quarries in the vicinity have undoubtedly destroyed potential habitat and subpopulations. The two subpopulations that remain, maintain distinct plastome compositions and these differences may have been initiated and/or enhanced by a reduction in gene flow via seeds as a result of habitat destruction between the remaining subpopulations. If human activity contributes to differences in observed population structure, the Phacelia example demonstrates that different forms of human disturbance can have different impacts on population structure. A similar case of differential effects of anthropomorphic disturbance occurred in Larix laricina, where a population that regenerated from a seed bank showed structure in allozyme loci, whereas a newly colonized field did not (Knowles et al., 1992).

\section{Temporal fluctuations}

Temporal variation in genetic structure may be shaped by at least two forces that do not influence spatial patterning: fluctuations in population size over time, and variation in seed output among plants. For each of these, an enhanced role for genetic drift is expected under uniparental inheritance, because of the expected reduction in $N_{\mathrm{e} \text {,mat }}$. The reduction in $N_{\mathrm{e} \text {,mat }}$ caused by 
differential seed production is analogous to the effects of unequal family size on $N_{\mathrm{e}}$ for biparentally inherited genes. In contrast to direct effects on temporal structure, differential seed output must be accompanied by limited seed dispersal to generate spatial differentiation.

Because of an annual habit, granite outcrop populations of $P$. dubia experience extreme year-to-year fluctuations in numbers. In the poorest growing years at EM-Rd, fewer than 30 plants could be found maturing seeds, compared to the most favourable years, when several hundred plants produce seeds (unpubl. data). In a Virginia population of $P$. dubia, a population crash reduced population size by three orders of magnitude, but complete recovery in numbers occurred within two years and there was no loss of allelic diversity (del Castillo, 1994). Despite the bottleneck, a seed bank that has been shown to maintain viable seeds for three to six years (Baskin \& Baskin, 1973) dampened temporal fluctuations in genotypic composition and maintained local patch structure through transient loss of most patches.

A highly skewed distribution of plant sizes within populations, with many small and few large plants, may further contribute to local cytoplasmic differentiation. In $P$. dubia, large plants produce hundreds of seeds whereas small ones often produce fewer than 20 seeds. Thus, unequal maternal contributions caused by differential seed production can result in preferential representation of plastome types from the larger plants. Because we have not assayed differential pollen success, it is premature to speculate whether the relative effects of differential reproduction through pollen and seeds is a primary factor causing different levels of population structure for maternal and biparentally inherited genes.

Chloroplast markers showed wide temporal fluctuations, most notably in the two populations (AP, EM$\mathrm{Rd}$ ) subject to severe anthropomorphic disturbance. The cause of the temporal effect at AP was a spike in the frequency of plastome D in 1990. However, sample sizes for collections prior to 1992 were relatively small, and conclusions from these data must be viewed with caution, because a temporal effect that may arise from genetic drift may also be inferred from sampling error associated with small sample sizes (Waples, 1989).

Of the four tests for nuclear temporal differentiation, one was significant (EM, GOT2) and another was marginally significant (KIO, GOT1). However, the most apparent temporal changes at these nuclear-encoded loci were in the presence and frequencies of rare alleles. At EM, a rare allele at GOT2 emerged in the 1992 sample and increased in frequency, in contrast to the loss of two rare alleles in the 1992 sample from KIO. Rare alleles were also subject to temporal fluctuations in the patchily distributed prairie species, Asclepias verticillata (Fore \&
Guttman, 1996). Although two of four allozyme loci showed significant temporal variation in a Virginia population of $P$. dubia, there was no loss of rare alleles and changes in allele frequencies were not correlated with a documented bottleneck. Loci of both nuclear and chloroplast genomes may be subject to temporal variation, but qualitative assessments of the relative impact of time on the two genomes should await more extensive intrapopulation sampling as well as data from more species.

We have presented evidence for spatial and temporal differentiation in biparentally and maternally inherited genes at both the local and global levels. Patterns of differentiation, most notably both stronger and more fine-scale structure for chloroplast genes, are consistent with expectations under a model of neutral markers experiencing genetic drift. If these inferences are correct, the underlying cause of the observation that three of the four localities surveyed maintain a plastome polymorphism remains elusive.

\section{References}

BASKIN, J. M. AND BASKIN, C. C. 1973. Delayed germination in seeds of Phacelia dubia var. dubia. Can. J. Bot., 51, 24812486.

BIRKY, C. W., JR. 1983. Relaxed cellular controls and organelle heredity. Science, 222, 468-475.

BONNIN, I., HUGUET, T., GHERARDI, M., PROSPERI, J.-M. AND OLIVIERI, I. 1996. High level of polymorphism and spatial structure in a selfing plant species, Medicago truncalata (Leguminosae), shown using RAPD markers. Am. J. Bot., 83, 843-855.

CAUJAPE-CASTElls, J. AND PedROlA-MONFORT, J. 1997. Spacetime patterns of genetic structure within a stand of Androcymbium gramineum (Cav.) McBride (Colchicaceae). Heredity, 79, 341-349.

CONSTANTINE, C. C., HOBBS, R. P. AND LyMBERY, A. J. 1994. FORTRAN programs for analyzing population structure from multilocus genotypic data. J. Hered., 84, 336-337.

DEL CASTILlo, R. F. 1994. Factors influencing the genetic structure of Phacelia dubia, a species with a seed bank and large fluctuations in population size. Heredity, 72, 446-458.

DESAlle, R., TEMPLETON, A., MORI, I., PLETSCHER, S. AND JOHNSON, J. S. 1987. Temporal and spatial heterogeneity of mtDNA polymorphism in natural populations of Drosophila mercatorum. Genetics, 116, 5-223.

ENNOS, R. A. 1994. Estimating the relative rates of pollen and seed migration among plant populations. Heredity, 72, 250259.

EPPERSON, B. K. 1990. Spatial autocorrelation of genotypes under directional selection. Genetics, 124, 757-771.

FORE, S. A. AND GUTTMAN, S. I. 1996. Spatial and temporal genetic structure of Asclepias verticillata (whorled milkweed) among prairie patches in a forested landscape. Can. J. Bot., 74, 1289-1297.

(C) The Genetical Society of Great Britain, Heredity, 82, 422-431. 
HANSEN, M. M. AND LOESCHCKE, v. 1996. Temporal variation in mitochondrial DNA haplotype frequencies in a brown trout (Salmo trutta L.) population that shows stability in nuclear allele frequencies. Evolution, 50, 454-457.

HEBERT, P. D. N. AND WARD, R. D. 1976. Enzyme variability in natural populations of Daphnia magna. IV. Ecological differentiation and frequency changes of genotypes at Audley End. Heredity, 36, 331-341.

HEYwOOD, J. S. 1991. Spatial analysis of genetic variation in plant populations. Ann. Rev. Ecol. Syst., 22, 335-355.

HONG, Y.-P., HIPKINS, v. D. AND STRAUSS, S. D. 1993. Chloroplast DNA diversity among trees, populations and species in the California closed-cone pines (Pinus radiata, Pinus muricata, Pinus attenuata). Genetics, 135, 1187-1196.

HOSSAERT-MCKEY, M., VALERO, M., MAGDA, D., JARRY, M., CUGUEN, J. AND VERNET, P. 1996. The evolving genetic history of a population of Lathyrus sylvestris: evidence from temporal and spatial genetic structure. Evolution, 50, 18081821.

IBRAHIM, K. M., NICHOLS, R. A. AND HEWITT, G. M. 1996. Spatial patterns of genetic variation generated by different forms of dispersal during range expansion. Heredity, 77, 282-291.

KNOWles, P., PERRY, P. J. AND FOSTER, H. A. 1992. Spatial genetic structure in two tamarack [Larix laricina (Du Roi) K. Koch] populations with differing establishment histories. Evolution, 46, 572-576.

LATTA, R. G. AND MITTON, J. B. 1997. A comparison of population differentiation across four classes of gene marker in limber pine (Pinus flexis James). Genetics, 146, 1153-1163.

LEONARDI, S. AND MENOZZI, P. 1996. Spatial structure of genetic variability in natural stands of Fagus sylvatica L. (beech) in Italy. Heredity, 77, 359-368.

LEVY, F. 1989. Mechanisms of Speciation in Phacelia. Ph.D. Dissertation, Duke University, Durham, NC.

LEVy, F., ANTONOVICS, J., BOYNTON, J. E. AND GILlHAM, N. W. 1996. A population genetic analysis of chloroplast DNA in Phacelia. Heredity, 76, 143-155.
MARUYAmA, T. AND FUeRst, P. A. 1984. Population bottlenecks and nonequilibrium models in population genetics. I. Allele numbers when populations evolve from zero variability. Genetics, 108, 745-763.

MCCAUlEY, D. E. 1994. Contrasting the distribution of chloroplast DNA and allozyme polymorphism among local populations of Silene alba: Implications for studies of gene flow in plants. Proc. Natl. Acad. Sci. U.S.A., 91, 8127-8131.

MCCAULEY, D. E. 1997. The relative contributions of seed and pollen movement to the local genetic structure of Silene alba. J. Hered., 88, 257-263.

McCAUley, D. E., STEVens, J. E., PERONI, P. A. AND RAVElli, J. A. 1996. The spatial distribution of chloroplast DNA and allozyme polymorphisms within a population of Silene alba (Caryophyllaceae). Am. J. Bot., 83, 727-731.

MILGROOM, M. G. AND LIPARI, S. E. 1995. Spatial analysis of nuclear and mitochondrial RFLP genotypes in populations of the chestnut blight fungus, Cryphonectaria parasitica. Mol. Ecol., 4, 633-642.

MILligAN, B. G. 1991. Chloroplast DNA diversity within and among populations of Trifolium pratense. Curr. Genet., 19, 411-416.

RAYMOND, M. AND ROUSSET, F. 1995a. GENEPOP (version 3.1): Population genetics software for exact tests and ecumenism. J. Hered., 86, 248-249.

RAYMOND, M. AND ROUSSET, F. 1995b. An exact test for population differentiation. Evolution, 49, 1280-1283.

SCHNEIDER, S., KUEFFER, J.-M., ROESSLI, D. AND EXCOFFIER, L. 1997. ARLEQUIN. A software for population genetic data analysis. Release 1.7. Genetics and Biometry Laboratory, University of Geneva, Switzerland.

VIARD, F., JUSTY, F. AND JARNE, P. 1997. Population dynamics inferred from temporal variation at microsatellite loci in the selfing snail Bulinus truncatus. Genetics, 146, 973-982.

WAPLES, R. S. 1989. Temporal variation in allele frequencies: testing the right hypothesis. Evolution, 43, 1236-1251. 